\title{
A Study of Cerebral Performance Categories Based on Initial Rhythm and Resuscitation Time Following In-Hospital Cardiac Arrest in a State Hospital in Turkey
}

\author{
Faruk Cicekci ${ }^{*}$, Ali Ulvi Uca ${ }^{2}$, Inci Kara ${ }^{1}$, Iskender Kara ${ }^{3}$ \\ 'Department of Anesthesiology, Medical Faculty, Selcuk University, Konya, Turkey \\ 2Department of Neurology, Necmettin Erbakan University, Medical Faculty, Konya, Turkey \\ ${ }^{3}$ Department of Anesthesiology, Konya State Numune Hospital, Konya, Turkey
}

*Corresponding Author: Faruk Cicekci, M.D., Assistant Professor, Department of Anesthesiology, Medical Faculty, Selcuk University, Konya, Turkey. Tel: +90-332-2415000, Email: farukcicekci@yahoo.com

Received November 23, 2017; Accepted February 5, 2018; Online Published April 21, 2018

\begin{abstract}
Background: The cerebral performance category (CPC) score is widely used in research and quality assurance to assess neurologic outcome following cardiac arrest. However, little is known about the results of the CPC in Turkey.

Objective: This study aimed to determine whether the CPC is associated with the initial rhythm and resuscitation time following resuscitation from in-hospital cardiac arrest.

Methods: This study compared the CPCs (CPC 1-2 and CPC 3-4-5) of patients discharged from the hospital after surviving cardiopulmonary arrest (CPA) during a 2-year period between June 2013 and June 2015 (at discharge, and at 6th, 12th, 18th, and 24th months) based on the initial rhythm (asystole/pulseless electrical activity and ventricular fibrillation/pulseless ventricular tachycardia) and resuscitation time (0-14 $\mathrm{min}$ and $15-30 \mathrm{~min}$ ) at the time of arrest.

Results: No difference was found between CPC 1-2 and CPC 3-4-5 scores at discharge or at 6th, 12th, 18th, and 24th months in terms of the first rhythm and resuscitation time $(P>0.05)$.

Conclusion: Patients discharged from the hospital following in-hospital cardiopulmonary resuscitation (CPR) were found to have no difference in 2-year CPC scores with respect to cardiac rhythms and resuscitation durations at the onset of resuscitation.
\end{abstract}

Keywords: Heart Arrest, Cardiopulmonary Resuscitation, Neurologic Examination

\section{Background}

Every year, hundreds of thousands of people are saved after heart and/or respiratory arrest (cardiopulmonary arrest [CPA] with cardiopulmonary resuscitation $[\mathrm{CPR}]) .{ }^{1}$ However, ischemia and subsequent reperfusion result in serious brain damage despite improvement in the cardiac function of patients. Unfortunately, a satisfactory improvement in brain function cannot be achieved in most cases. Therefore, successful resuscitation should be in the form of both heart and brain resuscitation. In this context, the best post-resuscitation measurement involves an assessment of brain function., ${ }^{2,3}$

The neurological condition varies between complete recovery and brain death., ${ }^{4,5}$ Various measures are available to evaluate brain function after resuscitation. The Utstein-style guidelines recommend the use of the cerebral performance category (CPC), a 5-point scale that tries to combine functional and cognitive domains to provide an assessment of brain healing. ${ }^{2,6,7}$ Several studies have demonstrated the effects of initial rhythms and resuscitation durations on CPC for short- or long-term survival and the rate of return of spontaneous circulation (ROSC) after CPR..$^{8-12}$

\section{Objective}

This study aimed to compare the CPC scores of patients during the 2-year post-discharge period based on the initial rhythm in the course of CPA (asystole/pulseless electrical activity [PEA]) and ventricular fibrillation (VF)/pulseless ventricular tachycardia (PVT) and the resuscitation period.

\section{Methods}

This case-control clinical study was conducted with 44 patients whose ROSC occurred through in-hospital

Copyright $\odot 2018$ The Author(s). This is an open-access article distributed under the terms of the Creative Commons Attribution License (http:// creativecommons.org/licenses/by/4.0), which permits unrestricted use, distribution, and reproduction in any medium, provided the original work is properly cited. 
CPR. The patients survived for at least 24 hours and were discharged after treatment in the Konya Numune Hospital between June 2013 and June 2015. Consent to conduct this study was obtained from the Selcuk University Faculty of Medicine Ethical Committee (Ref no: 2017-100). The CPC values of the studied patients were followed up for 2 years after discharge. Subjects who were younger than 18 years died before discharge from the hospital and, hence, were excluded from the study. The follow-up results are shown in Figure 1 as a flow chart.

In-hospital resuscitation for these patients was performed in accordance with current advanced life support protocols (2010 European Resuscitation Council Guideline). Demographic data, such as age and sex of the cases and clinical parameters related to $\mathrm{CPR}$, were recorded.

The information obtained from the doctors and patients' relatives was evaluated by the neurology specialist. Neurological status measurements were evaluated by the neurology specialist using the CPC, a 5-category scale

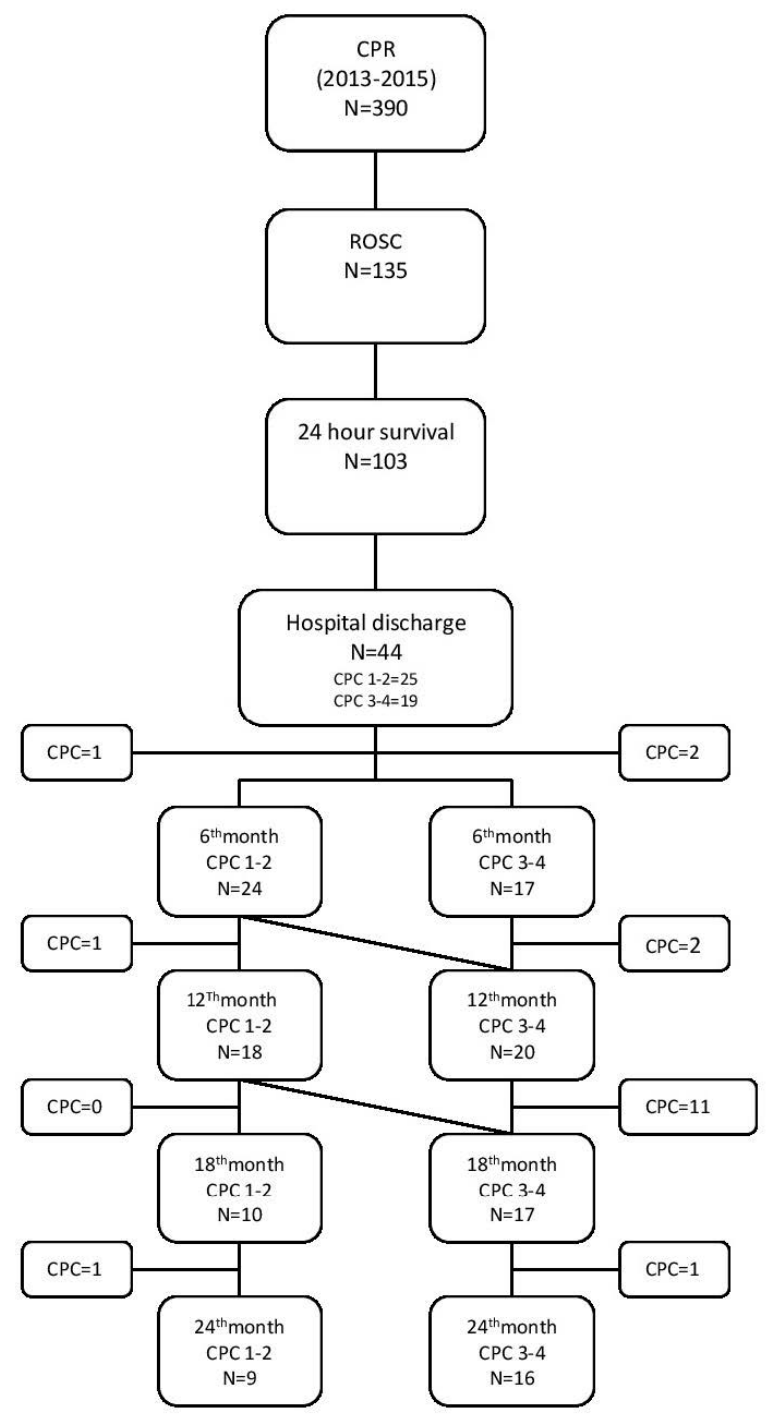

Figure 1. Flowchart of a Case-Control Clinical Study Follow-up. $\mathrm{CPR}$, cardiopulmonary resuscitation; CPC, cerebral performance category; ROSC, return of spontaneous circulation. based on the initial rhythm (asystole/pulseless electrical activity and ventricular fibrillation/pulseless ventricular tachycardia) and resuscitation time (0-14 minutes and 15-30 minutes) by calling patients or their relatives at the 6 th, 12th, 18th, and 24th months after discharge from the hospital. The 5 categories of the CPC are: CPC 1, conscious and alert with good cerebral performance; CPC 2, conscious and alert with moderate cerebral performance; CPC 3, conscious with severe cerebral disability; CPC 4, comatose or in persistent vegetative state; and CPC 5 , brain dead, circulation preserved. For those patients who did not give enough information, the follow-up information was obtained from the doctors who worked for homehealth services. CPC 1 and 2 were classified as favorable neurological outcomes, whereas CPC 3, 4, and 5 were evaluated as poor outcomes.

Analyses in the study were done using SPSS 20.0 software (SPSS, IL, USA). Continuous and categorical variables were presented in tables and graphs by subtracting descriptive measures. Continuous variables were expressed as mean \pm standard deviation (SD), whereas categorical variables were expressed as frequencies and percentage ratios. Cross tables were prepared, and the chi-square test was used to determine any relationships between categorical variables. A $P$ value (Fisher exact test) less than 0.05 was considered statistically significant for all analyses.

\section{Results}

This retrospective case-controlled study included 44 patients who were discharged and who survived 24 hours $(n=103)$ from ROSC $(n=135)$ from a total of 390 CPR patients between 2011 and 2013, and their neurological statuses were assessed between June 2013 and June 2015. The flow chart of the study is shown in Figure 1.

The socio-demographic and clinical values of the subjects are listed in Table 1. CPR starting time was 1.8 \pm 0.5 minutes. CPR was applied to 29 patients for $0-14$ minutes and to 15 patients for 15-30 minutes. No patients underwent CPR for 30 minutes or more. At the time of CPA, 10 patients had first rhythm asystole/PEA and 34 patients had VF/PVT. Moreover, 135 patients had the ROSC, and 103 patients survived for more than 24 hours.

During the course of this prospective clinical study, 44 patients survived and were discharged after successful CPR. At the time of discharge, the neurological evaluation scores of 10 patients (22.6\%) were CPC 1-2 and of 34 patients (77.4\%) were CPC 3-4 according to the initial rhythms in CPA asystole/NEA and VF/PVT, respectively.

At the time of discharge, the number of patients whose initial rhythms were asystole-PEA/VF-PVT in the course of CPA and their neurological evaluation scores $[n(\%)$, CPC 1-2; CPC 3-4; and CPC 5)] were 5 (11.3)/20 (45.5); 5 (11.3)/14 (31.9), and (Patients who survived after CPR but did not survive to discharge were not defined.), respectively. The difference between two groups ( asystolePEA/VF-PVT) was not statistically significant $(P=0.744)$.

At the sixth month of post-discharge, the number of 
Table 1. Demographic Information of 44 Study Subjects Who Survived to Hospital Discharge

\begin{tabular}{ll}
\hline & Study, N = 44 \\
\hline Gender, No. (\%) & $34(77.2)$ \\
Male & $10(22.8)$ \\
Female & $62.5(13.0)$ \\
Age, years (SD) & $1.8(0.5)$ \\
CPA arrival time (min) (SD) & $12.8(6.3)$ \\
Average CPR time (min) (SD) & \\
CPA occurrence time, No. (\%) & $23(52.3)$ \\
Weekday working days ${ }^{\mathrm{a}}$ & $9(20.4)$ \\
Weekday evenings/nights & \\
Weekend & \\
CPA arrival time, min (SD) & $12(27.3)$ \\
CPR time period (min), No. (\%) & $1.7(0.9)$ \\
0-14 & \\
15-30 & $29(65.9)$ \\
$>30$ & $15(34.1)$ \\
CPR initial rhythm, No. (\%) & $0(0)$ \\
Asystole/PEA & \\
VF/PVT & $10(22.8)$ \\
\hline
\end{tabular}

Abbreviations: SD, standard deviation; CPA, cardiopulmonary arrest; CPR, cardiopulmonary resuscitation; PEA, pulseless electrical activity; VF, ventricular fibrillation; PVT, pulseless ventricular tachycardia; CPC, cerebral performance category.

aMonday, Tuesday, Wednesday, Thursday, and Friday, between 08.00-16.00 h.

${ }^{\mathrm{b}}$ After working hours, between 16.00-08.00 h

cWeekends, between Friday 16.00 -Monday 08.00

patients [n (\%)] whose initial rhythms were asystole-PEA/ VF-PVT in the course of CPA and their neurological evaluation scores [n (\%), CPC 1-2; CPC 3-4; and CPC 5] were 4 (9.1)/20 (45.5); 3 (6.8)/14 (31.8); and 1 (2.29)/2 (4.5), respectively. The difference between 2 groups (asystolePEA/VF-PVT) was not statistically significant $(P=0.605)$.

At the 12th month, the number of patients whose initial rhythms were asystole/PEA in the course of CPA and their neurological evaluation scores [n (\%), CPC 1-2; CPC 3-4; and CPC 5] were 3 (7.3)/15 (36.6); 3 (7.3)/17 (41.5); and 1 (2.4)/2 (4.9), respectively. The difference between 2 groups was not statistically significant $(P=0.605)$.

At the 18th month, the number of patients whose initial rhythms were asystole-PEA/VF-PVT in the course of CPA and their neurological evaluation scores [n (\%), CPC 1-2; CPC 3-4; and CPC 5] were 2 (5.3)/8 (21.0); $2(5.3) / 15$ (39.5); and $0(0.0) / 11$ (28.9), respectively. No difference was found between 2 groups (asystole-PEA/VF-PVT) ( $P$ $=0.712$ ).

At the 24th month, the number of patients whose initial rhythms were asystole-PEA/VF-PVT in the course of CPA and their neurological evaluation scores [n (\%), CPC 1-2; CPC 3-4; and CPC 5] were 2 (7.4)/7 (25.9); 2 (7.4)/14 (51.9); and 1 (3.7)/1 (3.7), respectively. No difference was found between the 2 groups (asystole-PEA/VF-PVT) $(P=$ 0.712 ). The number of patients whose initial rhythms were asystole-PEA and their neurological evaluations are shown
Table 2. Number of Patients According to the Initial Rhythm in CPR and 2-Year Neurological Evaluation Scores

\begin{tabular}{llcrc}
\hline & & \multicolumn{3}{c}{ Initial Rhythm } \\
\cline { 3 - 5 } & CPC & Asystole/NEA & VF/PVT & P \\
\hline At discharge & $1-2$ & $5(11.3)$ & $20(45.5)$ & \\
N=44, No. (\%) & $3-4$ & $5(11.3)$ & $14(31.9)$ & 0.744 \\
& $5 *$ & $0(0)$ & $0(0)$ & \\
6th month & $1-2$ & $4(9.1)$ & $20(45.4)$ & \\
N=44, No. (\%) & $3-4$ & $3(6.8)$ & $14(31.8)$ & 0.783 \\
& 5 & $1(2.2)$ & $2(4.5)$ & \\
12th month & $1-2$ & $3(7.3)$ & $15(36.6)$ & \\
N=41, No. (\%) & $3-4$ & $3(7.3)$ & $17(41.5)$ & 0.605 \\
& 5 & $1(2.4)$ & $2(4.9)$ & \\
18th month & $1-2$ & $2(5.3)$ & $8(21.0)$ & \\
N=38, No. (\%) & $3-4$ & $2(5.3)$ & $15(39.5)$ & 0.712 \\
& 5 & $0(0.0)$ & $11(28.9)$ & \\
24th month & $1-2$ & $2(7.4)$ & $7(25.9)$ & \\
N=27, No. (\%) & $3-4$ & $2(7.4)$ & $14(51.9)$ & 0.807 \\
& 5 & $1(3.7)$ & $1(3.7)$ & \\
\hline Nenyyy & & & & \\
\hline
\end{tabular}

Neurological outcome assessed as cerebral performance category (CPC) at hospital discharge; data is presented as absolute numbers and relative frequencies

* Patients who survived after CPR but did not survive to discharge were not shown.

in Table 2.

$\mathrm{CPC}$ values of the patients according to the CPR time were followed up for 24 months after discharge. At the time of discharge, the number of patients who had 0 - to 14-minute and 15- to 30-minute resuscitation periods of $\mathrm{CPR}$ and their neurological evaluation scores [n (\%), CPC 1-2; CPC 3-4; and CPC 5] were 18 (40.9)/7 (15.9); 11 (25.0)/8 (18.2); and (Patients who survived after CPR but did not survive to discharge were not defined.), respectively. The difference between 2 groups (0- to 14-minute and 15to 30 -minute) was not statistically significant $(P=0.876)$.

At the sixth month post-discharge, the number of patients who had 0 - to 14 -minute and 15 - to 30 -minute resuscitation periods of $\mathrm{CPR}$ and their neurological evaluation scores [n (\%), CPC 1-2; CPC 3-4; and CPC 5] were 18 (40.9)/7 (15.9); 9 (20.5)/7 (15.9); and 2 (4.5)/1 (2.3), respectively. It appears there are three groups.

At the 12th month, the number of patients who had 0- to 14-min and 15- to 30-min resuscitation periods of CPR and their neurological evaluation scores [n (\%), CPC 1-2; CPC 3-4; and CPC 5] were 14 (34.2)/5 (12.2); 11 (26.8)/8 (19.5); and $2(4.9) / 1(2.5)$, respectively. The difference between two groups was not statistically significant $(P=0.657)$.

At the 18th month, the number of patients who had 0 to 14 -minute and 15- to 30-minute resuscitation periods of $\mathrm{CPR}$ and their neurological evaluation scores $[n(\%)$, CPC 1-2; CPC 3-4; and CPC 5] were 7 (18.4)/4 (10.5); $10(26.3) / 6(15.8)$ and $8(21.1) / 3(7.9)$, respectively. The difference between 2 groups was not statistically significant $(P=0.824)$.

At the 24th month, the number of patients who had 0 -to 14-minute and 15- to 30-minute resuscitation periods of 
CPR and their neurological assessment scores [n (\%), CPC 1-2; CPC 3-4; and CPC 5] were 6 (22.2)/4 (14.8); 9 (33.3)/6 (22.2) and $2(7.5) / 0(0.0)$, respectively. The difference between 2 groups ( 0 - to 14 -minute and 15 - to 30 -minute) was not significant $(P=0.563)$. Table 3 shows the number of patients who underwent resuscitation for 0-14 and 1530 minutes and their neurological evaluation scores based on the CPR duration.

\section{Discussion}

This study found that, when the resuscitation time and the initial cardiac rhythms during resuscitation were considered, long-term CPC scores did not differ in patients who were discharged from the hospital following in-hospital CPR.

It is well known that most patients who have cardiac resuscitation at the hospital cannot be discharged. ${ }^{14} \mathrm{~A}$ successful CPR depends on the presence of basic and advanced life support systems, the ability to perform early defibrillation, and the quality of CPR intervention. ${ }^{15}$ After an in-hospital cardiac arrest, survival at discharge is approximately $15 \%-20 \% .^{16,17}$ In Turkey, this rate is about $11 \%-25.7 \% .^{18,19}$

The debate on the most useful approach to assess the outcome of sudden cardiac arrest is ongoing. ${ }^{3}$ Although the purpose of the data and studies is balanced, the results of some studies indicate that CPC is a measure of longterm longevity and is useful for obtaining long-term resuscitation results. ${ }^{2,20}$ Moreover, these findings support the use of the CPC following neurological prognosis when post-arrest patients are discharged. For example, those classified as CPC 1-2 have shown better survival rates than those classified as CPC 3-4. The life expectancy of postarrest patients with different $\mathrm{CPC}$ scores during discharge is significantly different.

After discharge, long-term neurological results of survivors after a cardiac arrest are better than those with CPC scores of 1-2 and CPC scores of 3-5. That is, CPC 3 and CPC 4 scores are associated with worse long-term outcomes in patients after CPA. Thus, CPC scores may help in further studies to predict necessary controls and treatments of post-arrest patients after discharge from the hospital.

Although the discharge rate after an in-hospital CPA is approximately $18 \%$, arrest rhythm VF-VT is still slightly higher (36\%). ${ }^{16}$ The discharge rates of patients with the initial rhythm VF-PVT in different studies were $24 \%-$ $36 \%$. Goto et $\mathrm{al}^{9}$ showed that CPC 1-2 scores were $52.1 \%$ for VF-PVT rhythm and 18.2\% for asystole-PEA 1 month after discharge. In various other studies, CPC 1-2 scores at discharge were $26.5 \%-73 \%^{21-24}$ and CPC 3-4 scores were $0 \%-39.5 \% .{ }^{25}$ In the present study, the scores were CPC 1-2: 11.3\%, CPC 3-4: 11.3\%/CPC 1-2: 45.5\%, CPC 3-4: 31.9\% at the time of discharge (in the order of asystole-PEA/VFPVT). At the sixth month, CPC 1-2, CPC 3-4, and CPC 5 results were $(9.1 \% / 45.4 \%),(6.8 \% / 31.8 \%)$, and $(2.2 \% / 4.5 \%)$ (in the order of asystole-PEA/VF-PVT), respectively. At the
Table 3. Number of Patients Who Underwent Resuscitation Between 0-14 and 15-30 Minutes According to the CPR Period and 2-Year Neurological Evaluation Scores

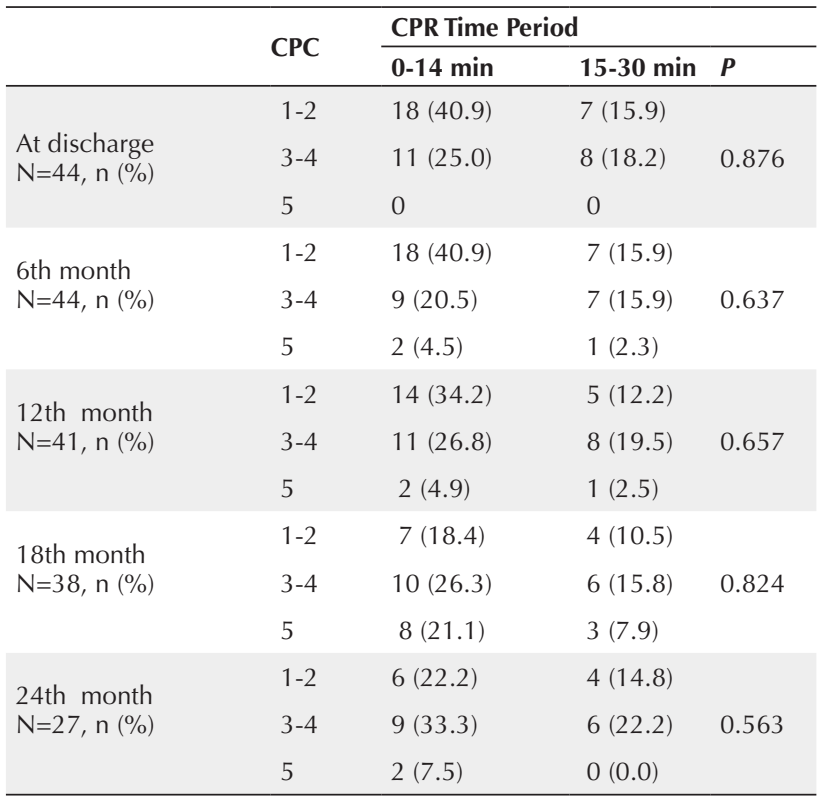

Neurological outcome assessed as cerebral performance category (CPC) at hospital discharge; Data is presented as absolute numbers and relative frequencies.

* Patients who survived after CPR but did not survive to discharge are not shown in Table 3.

12 th month, these scores were $(7.3 \% / 36.6 \%),(7.3 \% / 41.5 \%)$, and $(2.4 \% / 4.9 \%)$, respectively. At the 18 th month, they were $(5.3 \% / 21.0 \%),(5.3 \% / 39.5 \%)$, and $(0.0 \% / 28.9 \%)$, respectively. At the 24th month, they were $(7.4 \% / 25.9 \%)$, $(7.4 \% / 51.9 \%)$, and $(3.7 \% / 3.7 \%)$, respectively. Nielsen et $\mathrm{al}^{26}$ also showed that neurological performance does not change significantly in the majority of patients after cardiac arrest until 6 months after discharge. No significant change in CPC scores was observed during the first 6 months of the present study. However, although CPC 1-2 scores after 12 months were not statistically significant, a decrease in good neurological markers and an increase in mortality rates were noted.

Moreover, the changes in CPC scores were not statistically significant according to the time periods in this study, but more changes were observed from the 12 th month to the 18 th month.

In another clinical trial, VF was shown to reduce shortand long-term survival rates significantly when not treated for more than 10 minutes. ${ }^{20}$ In animal models, VF has been shown to reduce short- and long-term neurological recovery when not treated for longer than 12-13 minutes. ${ }^{27}$ In the present study, CPR times (according to 0-14 min/15-30 min order) were CPC 1-2: 40.9\%/15.9\% and CPC 3-4: $25.0 \% / 18.2 \%$, respectively.

CPC 1-2; CPC 3-4; and CPC 5 scores (according to 0-14 min/15-30 min order) were (40.9\%/15.9\%); (20.5\%/15.9\%); and $(4.5 \% / 2.3 \%)$, respectively, in the sixth month. At the 12 th month, they were $(34.2 \% / 12.2 \%) ;(26.8 \% / 19.5 \%)$; and $(4.9 \% / 2.5 \%)$, respectively. At the 18 th month, they were $(18.4 \% / 10.5 \%) ;(26.3 \% / 15.8 \%)$; and $(21.1 \% / 7.9 \%)$, 
respectively. At the 24th month, they were (22.2\%/14.8\%); $(33.3 \% / 22.2 \%)$; and $(7.5 \% / 0.0 \%)$, respectively.

Particularly after 12 months, a statistically insignificant decrease in the CPC 1-2 score and increase in the mortality rate were seen in patients who were resuscitated for 0-14 minutes in the present study.

This novel study involved the longest neurological follow-up periods of patients who survived CPA and were discharged from the hospital.

It is believed that the decrease in neurological performance after the 12th month in patients who had the initial rhythms of VF-PVT and better resuscitation times (e.g., 0-14 minutes) may be related to the treatmentrelated disorders of their primary diseases, lack of home care, or the appearance of new diseases.

The current study had some limitations. First, although the study was a prospective clinical study, only 2-year-old data from a single center was used. Hence, the lack of a study population prevented conducting more valuable statistical studies. Second, the concept of therapeutic hypothermia could not be fully applied to CPA patients when the study was planned and performed. Third, primary disease follow-ups of the patients could not be done after their discharge. Fourth, the CPC scoring system was used in the study; however, some other criteria could be used as well. Fifth, since most studies have been performed on outhospital cardiac arrest cases, the number of neurological outcomes of in-hospital cardiac arrests after discharge in the literature was insufficient.

\section{Conclusion}

The survivors of cardiac arrest were found to have deteriorated neurological outcomes after discharge during a 2-year period in this study. These results did not depend on the duration of arrest or the first rhythm and resuscitation period. The present results were contrary to the findings of existing studies. It suggested that the postdischarge follow-up and treatments of the patients were not enough. Therefore, it is recommended that adequate training be given to the families or the caregivers of patients. It is thought that the provision of pre-discharge palliative care services to patients with the help of health care professionals may improve the neurological results after discharge.

\section{Authors' Contributions}

Concept: FC; Design: FC, IK; Supervision: IK, HK; Funding: FC; Materials: FC; Data collection and/or processing: FC; Analysis and/or interpretation: AUU; Literature review: FC; Writing: FC, IK, AUU; Critical review: HK.

\section{Conflict of Interest Disclosures}

The authors declare that they have no conflicts of interest.

\section{Ethical Approval}

Informed consent was obtained from patients and patients' relatives who participated in this study. This study was

\section{Research Highlights}

\section{What Is Already Known?}

Even if heart functions improve, in most patients, returning to life with cardiopulmonary resuscitation $(\mathrm{CPR})$, ischemia, and subsequent reperfusion causes serious brain damage. In this context, the best postresuscitation measurement includes an assessment of brain function.

\section{What This Study Adds?}

This study found that when the resuscitation time and the initial cardiac rhythms during resuscitation were considered, long-term CPC scores did not differ in patients who were discharged from the hospital following an in-hospital CPR in Turkey.

registered in the Australian New Zealand Clinical Trial Registry ACTRN under Trial ID: ACTRN12617001370392.

\section{References}

1. Deem S, Hurford WE. Respiratory controversies in the critical care setting. Should all patients be treated with hypothermia following cardiac arrest? Respir Care. 2007;52(4):443-450; discussion 450-441.

2. Jacobs I, Nadkarni V, Bahr J, et al. Cardiac arrest and cardiopulmonary resuscitation outcome reports: update and simplification of the Utstein templates for resuscitation registries: a statement for healthcare professionals from a task force of the International Liaison Committee on Resuscitation (American Heart Association, European Resuscitation Council, Australian Resuscitation Council, New Zealand Resuscitation Council, Heart and Stroke Foundation of Canada, InterAmerican Heart Foundation, Resuscitation Councils of Southern Africa). Circulation. 2004;110(21):3385-3397. doi:10.1161/01. cir.0000147236.85306.15.

3. Becker LB, Aufderheide TP, Geocadin RG, et al. Primary outcomes for resuscitation science studies: a consensus statement from the American Heart Association. Circulation. 2011;124(19):21582177. doi:10.1161/CIR.0b013e3182340239.

4. Longstreth WT Jr, Inui TS, Cobb LA, Copass MK. Neurologic recovery after out-of-hospital cardiac arrest. Ann Intern Med. 1983;98(5 Pt 1):588-592. doi:10.7326/0003-4819-98-5-588.

5. Moulaert VR, Verbunt JA, van Heugten CM, Wade DT. Cognitive impairments in survivors of out-of-hospital cardiac arrest: a systematic review. Resuscitation. 2009;80(3):297-305. doi:10.1016/j.resuscitation.2008.10.034

6. A randomized clinical trial of calcium entry blocker administration to comatose survivors of cardiac arrest. Design, methods, and patient characteristics. The Brain Resuscitation Clinical Trial II Study Group. Control Clin Trials. 1991;12(4):525545. doi: 10.1016/0197-2456(91)90011-A.

7. Mild therapeutic hypothermia to improve the neurologic outcome after cardiac arrest. N Engl J Med. 2002;346(8):549556. doi:10.1056/NEJMoa012689.

8. Neumar RW, Otto CW, Link MS, et al. Part 8: adult advanced cardiovascular life support: 2010 American Heart Association Guidelines for Cardiopulmonary Resuscitation and Emergency Cardiovascular Care. Circulation. 2010;122(18 Suppl 3):S729767. doi:10.1161/circulationaha.110.970988.

9. Goto Y, Maeda T, Nakatsu-Goto Y. Neurological outcomes in patients transported to hospital without a prehospital return of spontaneous circulation after cardiac arrest. Crit Care. 2013;17(6):R274. doi:10.1186/cc13121 
10. Hogler S, Sterz F, Sipos W, et al. Distribution of neuropathological lesions in pig brains after different durations of cardiac arrest. Resuscitation. 2010;81(11):1577-1583. doi:10.1016/j. resuscitation.2010.07.005.

11. Janata A, Bayegan K, Sterz F, et al. Limits of conventional therapies after prolonged normovolemic cardiac arrest in swine. Resuscitation. 2008;79(1):133-138. doi:10.1016/j. resuscitation.2008.04.005

12. Janata A, Weihs W, Schratter A, et al. Cold aortic flush and chest compressions enable good neurologic outcome after 15 mins of ventricular fibrillation in cardiac arrest in pigs. Crit Care Med. 2010;38(8):1637-1643. doi:10.1097/CCM.0b013e3181e78b9a.

13. Safar P. Resuscitation after Brain Ischemia. In: Grenvik A, Safar $\mathrm{P}$, eds. Brain Failure and Resuscitation. New York: Churchill Livingstone; 1981:155-184.

14. van Walraven C, Forster AJ, Parish DC, et al. Validation of a clinical decision aid to discontinue in-hospital cardiac arrest resuscitations. JAMA. 2001;285(12):1602-1606. doi:10.1001/ jama.285.12.1602

15. Tok D, Keles GT, Toprak V, Topcu I. Assessment of in-hospital cardiopulmonary resuscitation using Utstein template in a university hospital. Tohoku J Exp Med. 2004;202(4):265-273. doi:10.1620/tjem.202.265.

16. Nadkarni VM, Larkin GL, Peberdy MA, et al. First documented rhythm and clinical outcome from in-hospital cardiac arrest among children and adults. JAMA. 2006;295(1):50-57. doi:10.1001/jama.295.1.50.

17. Sandroni C, Nolan J, Cavallaro F, Antonelli M. In-hospital cardiac arrest: incidence, prognosis and possible measures to improve survival. Intensive Care Med. 2007;33(2):237-245. doi:10.1007/ s00134-006-0326-z.

18. Cicekci F, Sizer C, Geredeli C, Karaca H, Kara F, Karaibrahimoglu A. Comparison of in-Hospital Cardiopulmonary Resuscitation Results Before and After the Introduction Code Blue. Turkiye Klinikleri J Anest Reanim. 2015;13(2):78-85. doi:10.5336/ anesthe.2014-42431.

19. Oguzturk H, Turtay MG, Tekin YK, Sarihan E. Cardiac arrests in the emergency ward and our experiences on the cardiopulmonary resuscitation. Kafkas J Med Sci. 2011;1(3):114-117. doi:10.5505/ kjms.2011.08108.

20. Phelps R, Dumas F, Maynard C, Silver J, Rea T. Cerebral Performance Category and long-term prognosis following out-ofhospital cardiac arrest. Crit Care Med. 2013;41(5):1252-1257. doi:10.1097/CCM.0b013e31827ca975.

21. Storm C, Nee J, Krueger A, Schefold JC, Hasper D. 2-year survival of patients undergoing mild hypothermia treatment after ventricular fibrillation cardiac arrest is significantly improved compared to historical controls. Scand J Trauma Resusc Emerg Med. 2010;18:2. doi:10.1186/1757-7241-18-2.

22. Kim YS, Lee YJ, Won KB, et al. Extracorporeal Cardiopulmonary Resuscitation with Therapeutic Hypothermia for Prolonged Refractory In-hospital Cardiac Arrest. Korean Circ J. 2017;47(6):939-948. doi:10.4070/kcj.2017.0079.

23. Hsu CH, Li J, Cinousis MJ, et al. Cerebral performance category at hospital discharge predicts long-term survival of cardiac arrest survivors receiving targeted temperature management. Crit Care Med. 2014;42(12):2575-2581. doi:10.1097/ ccm.0000000000000547.

24. Reis AG, Nadkarni V, Perondi MB, Grisi S, Berg RA. A prospective investigation into the epidemiology of in-hospital pediatric cardiopulmonary resuscitation using the international Utstein reporting style. Pediatrics. 2002;109(2):200-209. doi:10.1542/ peds.109.2.200.

25. Agarwal S, Presciutti A, Roth W, et al. Determinants of LongTerm Neurological Recovery Patterns Relative to Hospital Discharge Among Cardiac Arrest Survivors. Crit Care Med. 2018;46(2):e141-e150. doi:10.1097/ccm.0000000000002846.

26. Nielsen N, Hovdenes J, Nilsson F, et al. Outcome, timing and adverse events in therapeutic hypothermia after out-of-hospital cardiac arrest. Acta Anaesthesiol Scand. 2009;53(7):926-934. doi:10.1111/j.1399-6576.2009.02021.x.

27. Laver S, Farrow C, Turner D, Nolan J. Mode of death after admission to an intensive care unit following cardiac arrest. Intensive Care Med. 2004;30(11):2126-2128. doi:10.1007/ s00134-004-2425-z. 